\title{
Coefficient Estimates and Fekete- Szegö Inequality for a Subclass of Bi-Univalent Functions Defined by Symmetric Q-Derivative Operator by Using Faber Polynomial Techniques
}

\author{
G. Saravanan ${ }^{1}$, Muthunagai. $\mathbf{K}^{2}$ \\ ${ }^{1,2}$ School of Advanced Sciences, VIT University, Chennai - 600 127, Tamil Nadu, India. \\ ${ }^{1}$ Present Address: Department of Mathematics, Patrician College of Arts and Science, Adyar, \\ Chennai-600020, Tamil Nadu, India.
}

\begin{abstract}
Article Info
Article history:

Received Jan $12^{\text {th }}, 2018$

Revised Apr $20^{\text {th }}, 2018$

Accepted May $26^{\text {th }}, 2018$

\section{Keyword:}

Bi-univalent

Faber Polynomials

Symmetric Q-Derivative

Fekete- Szegö problem
\end{abstract}

\section{Correspondng Author:}

G. Saravanan,

School of Advanced Sciences, VIT University,

Chennai - 600 127, Tamil Nadu, India.

Present Address: Department of Mathematics,

Patrician College of Arts and Science, Adyar,

Chennai-600020, Tamil Nadu, India.

Email: gsaran825@yahoo.com

\section{Introduction}

Let $\mathrm{A}$ be the class of all normalized functions of the form

$$
f(z)=z+\sum_{n=2}^{\infty} a_{n} z^{n}
$$

Which are analytic in the Unit disk U.

A function that is regular (holomorphic) in $U$ is said to be univalent in $U$ if it assumes no value more than once in $\mathrm{U}$. Denote by $\mathrm{S}$, the subclass of $\mathrm{A}$, of all univalent functions in $\mathrm{U}$.

For $f(\mathrm{z})$ and $g(\mathrm{z})$ analytic in $\mathrm{U}$, we say that $f(\mathrm{z})$ is subordinate to $g(\mathrm{z})$, written, $f(\mathrm{z}) \prec \mathrm{g}(\mathrm{z})$, if there exists a Schwarz function $w(\mathrm{z})$ with $w(\mathrm{z})=0$ and $|\mathrm{w}(\mathrm{z})|<1$ in $\mathrm{U}$ such that $f(\mathrm{z})=\mathrm{g}(\mathrm{w}(\mathrm{z}))$. That is if the range of one holomorphic function is contained in that of the second and these functions agree at a single point, then a sharp comparison of these two functions can be made.

The problem of finding sharp bounds for the linear functional $\left|a_{3}-\zeta a_{2}^{2}\right|$ of any compact family of functions is popularly known as the Fekete-Szegó problem. This coefficient functional on the normalized analytic 
functions in the unit disk represents various geometric quantities. For example, for $\zeta=1$, the functional represents Schwarzian derivative, which plays a significant role in the theory of univalent functions, conformal mapping and hypergeometric functions.

A function $f(\mathrm{z}) \in \mathrm{A}$ is said to be bi-univalent in $\mathrm{U}$, if $f(\mathrm{z}) \in S$ and its inverse has an analytic continuation to $|\mathrm{w}|<1$. The class of all bi-univalent functions is denoted by $\Sigma$.

The concept of bi-univalent functions was introduced by Lewin [18] who proved that if $f(\mathrm{z})$ is bi-univalent, then $\left|a_{2}\right|<1.51$. Brannan and Clunie [10] improved Lewin's result to $\left|a_{2}\right| \leq \sqrt{2}$. There is a rich literature on the estimates of the initial coefficients of bi-univalent functions (see $[11,13,16,24,25,26]$ ). However not much is known about the estimates of higher coefficients. It is well known that every function $f \in S$ has an inverse $f^{-1}$, satisfying $f^{-1}(f(z))=z,(z \in U)$ and $f\left(f^{-1}(w)\right)=w,\left(|| w<r_{0}(f) ; r_{0}(f) \geq \frac{1}{4}\right)$, where

$$
f^{-1}(w)=w-a_{2} w^{2}+\left(2 a_{2}^{2}-a_{3}\right) w^{3}-\left(5 a_{2}^{3}-5 a_{2} a_{3}+a_{4}\right) w^{4}+\cdots
$$

Let $\mathrm{K}$ be simply connected, compact set in the Complex plane. Let $h$ be analytic on K. It is possible to approximate $h$ by polynomials uniformly on K called Faber polynomials, introduced by Faber [12]. These polynomials play an important role in geometric function theory.

A detailed discussion about Faber polynomial expansion for functions $f \in S$ of the form has been carried out in [[1], [2], [3]].

Geometric function theory provides a platform to have a multiple dimensional view on the different subclasses of analytic functions with help of q- calculus which is an effective tool of investigation. For example, the theory of q- calculus is used to describe the extension of the theory of univalent functions. For basic definitions, applications, terminologies, geometric properties and approximation one can refer [[5], [8], [9], [14], [17], [19], [20], [21]]. Let us suppose $0<q<1$ throughout this paper.

\section{Definition. 1}

The $q$-derivative of a function $\mathrm{f}$ is defined on a subset of $\square$ is given by

and $\left(D_{q} f\right)(0)=f^{\prime}(0)$ provided $f^{\prime}(0)$ exists.

$$
\left(D_{q} f\right)(z)=\frac{f(z)-f(q z)}{(1-q) z}, \text { if } z \neq 0
$$

Note that

$$
\lim _{q \rightarrow 1^{-}}\left(D_{q} f\right)(z)=\lim _{q \rightarrow 1^{-}} \frac{f(z)-f(q z)}{(1-q) z}=f^{\prime}(z)
$$

if $f$ is differentiable. From (1), we have

$$
\left(D_{q} f\right)(z)=1+\sum_{n=2}^{\infty}[\mathrm{n}]_{q} a_{n} z^{n-1}
$$

Where the symbol $[n]_{q}$ denotes the number

\section{Definition. 2}

$$
[n]_{q}=\frac{1-q^{n}}{1-q}
$$

The symmetric $q$-derivative $\tilde{D}_{q} f$ of a function $f$ given by (1) is defined as follows: 


$$
\left(\tilde{D}_{q} f\right)(z)=\frac{f(q z)-f\left(q^{-1} z\right)}{\left(q-q^{-1}\right) z}, \quad \text { if } z \neq 0,
$$

and $\left(\tilde{D}_{q} f\right)(0)=f^{\prime}(0)$ provided $f^{\prime}(0)$ exists.

From (3), we have the deduction

$$
\left(\tilde{D}_{q} f\right)(z)=1+\sum_{n=2}^{\infty}[n]_{q} a_{n} z^{n-1},
$$

Where the symbol $[n]_{q}$ denotes the number

From (2) and (4), we also have

$$
[n]_{q}=\frac{q^{n}-q^{-n}}{q-q^{-1}}
$$

$$
\begin{aligned}
\left(\tilde{D}_{q} g\right)(w) & =\frac{g(q w)-g\left(q^{-1} w\right)}{\left(q-q^{-1}\right) w} \\
& =1-[2]_{q} a_{2} w+[3]_{q}\left(2 a_{2}^{2}-a_{3}\right) w^{2}-[4]_{q}\left(5 a_{2}^{3}-5 a_{2} a_{3}+a_{4}\right) w^{3}+\cdots
\end{aligned}
$$

Lemma. $1[6,22]$

If the function $p \in P$ is defined by

$$
p(z)=1+p_{1} z+p_{2} z^{2}+p_{3} z^{3}+\cdots
$$

then

$$
\left|p_{n}\right| \leq 2(n \in \square=\{1,2,3, \cdots\}),
$$

and

$$
\left|p_{2}-\frac{p_{1}^{2}}{2}\right| \leq 2-\frac{\left|p_{1}\right|^{2}}{2}
$$

Let $\varphi$ be an analytic function with positive real part in $\mathrm{U}$, with $\varphi(0)=1$ and $\varphi^{\prime}(0)>0$. Also, let $\varphi(U)$ be starlike with respect to 1 and symmetric with respect to the real axis. Then, $\varphi$ has the Taylor series expansion

$$
\varphi(z)=1+B_{1} z+B_{2} z^{2}+B_{3} z^{3}+\cdots\left(B_{1}>0\right) \text {. (6) }
$$

\section{Main Results}

\section{Definition. 3}

Let $f \in A$. Then $f \in \mathrm{R}_{\Sigma}(b, q, \varphi), b \in \square-\{0\}$ if $f \in \Sigma$,

and

$$
\operatorname{Re}\left(1+\frac{1}{b}\left(\left(D_{q} f\right)(z)-1\right)\right) \prec \varphi(u)(7)
$$

$$
\operatorname{Re}\left(1+\frac{1}{b}\left(\left(D_{q} g\right)(w)-1\right)\right) \prec \varphi(v)(8)
$$

Where $g=f^{-1}$.

\subsection{COEFFICIENT BOUNDS FOR FUNCTIONS BELONGING TO THE CLASS $\operatorname{R}_{\Sigma}(b, q, \varphi)$}

\section{Theorem 1}

Let $f \in \mathrm{R}_{\Sigma}(b, q, \varphi)$ and $g=f^{-1} \in \mathrm{R}_{\Sigma}(b, q, \varphi)$. If $a_{k}=0$ for $2 \leq k \leq n-1$ then

$$
a_{n} \leq \frac{2 b}{[n]_{q}} ; n \geq 3 \text {. }
$$




\section{Proof}

Let $f \in \mathrm{R}_{\Sigma}(b, q, \varphi)$ and $\varphi \in \mathrm{P}$, then there exists two Schwarz functions $u(z)=c_{1} z+c_{2} z^{2}+\cdots \quad$ and $v(w)=d_{1} w+d_{2} w^{2}+\cdots$ such that

$$
\begin{gathered}
1+\frac{1}{b}\left(\left(D_{q} f\right)(z)-1\right)=\varphi(u(z))(9) \\
1+\frac{1}{b}\left(\left(D_{q} g\right)(w)-1\right)=\varphi(v(w))(10)
\end{gathered}
$$

Where

$$
\varphi(u(z))=1+\sum_{n=1}^{\infty} \sum_{k=1}^{n} \varphi_{k} D_{n}^{k}\left(c_{1}, c_{2}, \cdots c_{n}\right) z^{n}
$$

and

$$
\varphi(v(w))=1+\sum_{n=1}^{\infty} \sum_{k=1}^{n} \varphi_{k} D_{n}^{k}\left(d_{1}, d_{2}, \cdots d_{n}\right) w^{n}
$$

From (9) and (11) we have

$$
\frac{[n]_{q} a_{n}}{b}=\sum_{k=1}^{n-1} \varphi_{k} D_{n}^{k}\left(c_{1}, c_{2}, \cdots c_{n}\right), n \geq 2 .
$$

From (10) and (12), we have

$$
\frac{[n]_{q} b_{n}}{b}=\sum_{k=1}^{n-1} \varphi_{k} D_{n}^{k}\left(d_{1}, d_{2}, \cdots d_{n}\right), n \geq 2 .
$$

For $a_{k}=0$ for $2 \leq k \leq n-1$, (13) and (14) respectively yield

and

$$
\frac{[n]_{q} a_{n}}{b}=\varphi_{1} c_{n-1}
$$

$$
\frac{[n]_{q} b_{n}}{b}=-\frac{[n]_{q} a_{n}}{b}=\varphi_{1} d_{n-1}
$$

By definition of $K_{n}^{p}$ we have $b_{n}=-a_{n}$.

Upon simplification, we obtain

$$
\begin{gathered}
a_{n}=\frac{b}{[n]_{q}} \varphi_{1} c_{n-1}(15) \\
a_{n}=-\frac{b}{[n]_{q}} \varphi_{1} d_{n-1}(16)
\end{gathered}
$$

Taking the absolute values of (15) and (16) and using the facts that $\left|\varphi_{1}\right| \leq 2,\left|c_{n-1}\right| \leq 1$ and $\left|d_{n-1}\right| \leq 1$, we obtain

\section{Remark 1}

$$
a_{n} \mid \leq \frac{2 b}{[n]_{q}}
$$

If $q \rightarrow 1^{-}$then the above theorem reduces to the results of Hamidi and Jahangiri [15].

\section{Remark 2}

If $b=1$ then above theorem reduces to the results of Altinkaya and Yalcin [7] (Theorem 7 for $\mathrm{p}=1$ ). 


\section{Theorem 2}

Let $f \in \mathrm{R}_{\Sigma}(b, q, \varphi)$ and $g=f^{-1} \in \mathrm{R}_{\Sigma}(b, q, \varphi)$. Then

$$
\begin{gathered}
\text { (i) } a_{2} \mid \leq\left\{\begin{array}{l}
\frac{2 d b}{q^{2}+1}, \quad \mid b<\frac{q^{4}+2 q^{2}+1}{q^{4}+q^{2}+1} \\
\frac{2 q \sqrt{b}}{\sqrt{q^{4}+q^{2}+1}}, \quad \mid \quad b \geq \frac{q^{4}+2 q^{2}+1}{q^{4}+q^{2}+1} .
\end{array}\right. \\
\text { (ii) } a_{3} \mid \leq\left\{\begin{array}{l}
\frac{4 q^{2} \mid b^{2}}{\left(q^{2}+1\right)^{2}}+\frac{2 q^{2}|b|}{q^{4}+q^{2}+1}, \quad|b|<\frac{\left(q^{2}+1\right)^{2}}{2\left(q^{4}+q^{2}+1\right)} \\
\frac{4 q^{2} \mid b}{q^{4}+q^{2}+1}, \quad \mid b \geq \frac{\left(q^{2}+1\right)^{2}}{2\left(q^{4}+q^{2}+1\right)} .
\end{array}\right. \\
\text { (iii) } a_{3}-\mu a_{2}^{2} \leq \frac{2 \mu q^{2} b}{q^{4}+q^{2}+1}, \mu=1, \frac{3}{2}, 2 .
\end{gathered}
$$

\section{Proof}

Letting $n=2$ and $n=3$ in [13] and [14] respectively, we get

$$
\frac{[2]_{q} a_{2}}{b}=\varphi_{1} c_{1}
$$

$$
\frac{[3]_{q} a_{3}}{b}=\varphi_{1} c_{2}+\varphi_{2} c_{1}^{2}
$$

and

$$
\begin{gathered}
\frac{[2]_{q} b_{2}}{b}=\varphi_{1} d_{1}(19) \\
\frac{[3]_{q} b_{3}}{b}=\varphi_{1} d_{2}+\varphi_{2} d_{1}^{2}(20)
\end{gathered}
$$

Comparing (5) with (19) and (20)

$$
\begin{gathered}
\frac{-[2]_{q} a_{2}}{b}=\varphi_{1} d_{1}(21) \\
\frac{[3]_{q}\left(2 a_{2}^{2}-a_{3}\right)}{b}=\varphi_{1} d_{2}+\varphi_{2} d_{1}^{2}
\end{gathered}
$$

Using $c_{1}=-d_{1}$ in either of (17) and (21), we deduce

From (18) and (22), we get

$$
\left|a_{2}\right| \leq \frac{2|b|}{[\tilde{2}]_{q}}=\frac{2 q|b|}{q^{2}+1}
$$

$$
\frac{2[3]_{q} a_{2}^{2}}{b}=\varphi_{1}\left(c_{2}+d_{2}\right)+\varphi_{2}\left(c_{1}^{2}+d_{1}^{2}\right)
$$

and thus 


$$
\left|a_{2}\right| \leq \sqrt{\frac{4|b|}{[3]_{q}}}=\frac{2 q \sqrt{|b|}}{\sqrt{q^{4}+q^{2}+1}}
$$

Now the bounds for $\left|a_{2}\right|$ are justified since $\left|a_{2}\right| \leq \sqrt{\frac{4|b|}{[3]_{q}}}=\frac{2 q \sqrt{|b|}}{\sqrt{q^{4}+q^{2}+1}}$ for $|b|<\frac{q^{4}+2 q^{2}+1}{q^{4}+q^{2}+1}$.

From (18), we get

$$
\left|a_{3}\right|=\frac{|b|\left(\left|\varphi_{1} c_{2}+\varphi_{2} c_{1}^{2}\right|\right)}{[3]_{q}} \leq \frac{4|b|}{[3]_{q}}=\frac{4 q^{2}|b|}{q^{4}+q^{2}+1}
$$

On the other hand subtracting (22) from (18).

$$
\frac{2[3]_{q}}{b}\left(a_{3}-a_{2}^{2}\right)=\varphi_{1}\left(c_{2}-d_{2}\right)
$$

Solving the above equation for $a_{3}$ and taking absolute value

$$
\left|a_{3}\right| \leq \frac{4 q^{2}|b|^{2}}{\left(q^{2}+1\right)^{2}}+\frac{2 q^{2}|b|}{q^{4}+q^{2}+1}
$$

Now, Theorem 2.2 (ii) follows from (23) and (25) upon noticing that

For the third part of the theorem, we rewrite (24) as

$$
\frac{4 q^{2}|b|^{2}}{\left(q^{2}+1\right)^{2}}+\frac{2 q^{2}|b|}{q^{4}+q^{2}+1}<\frac{4|b| q^{2}}{q^{4}+q^{2}+1} \text { if }|b|<\frac{\left(q^{2}+1\right)^{2}}{2\left(q^{4}+q^{2}+1\right)}
$$

$$
a_{3}-a_{2}^{2}=\frac{b}{2[3]_{q}}\left(\varphi_{1}\left(c_{2}-d_{2}\right)\right)
$$

Taking absolute values, we get

We rewrite (22) as

$$
\left|a_{3}-a_{2}^{2}\right|=\frac{|b|\left|\varphi_{1}\left(c_{2}-d_{2}\right)\right|}{2[3]_{q}} \leq \frac{2|b| q^{2}}{q^{4}+q^{2}+1}
$$

$$
2 a_{2}^{2}-a_{3}=\frac{b}{[3]_{q}}\left(\varphi_{1} d_{2}+\varphi_{2} d_{1}^{2}\right)
$$

Taking absolute values, we get

$$
\left|a_{3}-2 a_{2}^{2}\right|=\frac{|b| q^{2}}{q^{4}+q^{2}+1}\left|\varphi_{1} d_{2}+\varphi_{2} d_{1}^{2}\right| \leq \frac{4|b| q^{2}}{q^{4}+q^{2}+1}
$$

Adding (26) and (27) and taking absolute value,

$$
\left|a_{2}-\frac{3}{2} a_{2}^{2}\right| \leq \frac{3|b| q^{2}}{q^{4}+q^{2}+1} .
$$

\subsection{FEKETE- SZEG $\ddot{O}$ INEQUALITY FOR FUNCTIONS BELONGING TO THE CLASS $\mathrm{R}_{\Sigma}(b, q, \varphi)$}

\section{Theorem 3}

Let $f$ given by (1) be in the class $\mathrm{R}_{\Sigma}(b, q, \varphi)$ and $\zeta \in \square$. Then 


$$
a_{3}-\zeta a_{2}^{2} \mid \leq \begin{cases}\frac{B_{1} \mid b}{4[3]_{q}} & \text { for } 0 \$ h(\zeta) \leq \frac{1}{4[3]_{q}} \\ 4 B_{1} \mid h(\zeta) & \text { for } h(\zeta) \geq \frac{1}{4[3]_{q}} .\end{cases}
$$

where $h(\zeta)=\frac{B_{1}^{2}(1-\zeta)}{4\left[b[3]_{q} B_{1}^{2}+[2]_{q}^{2}\left(B_{1}-B_{2}\right)\right]}$

\section{Proof}

Let $f \in \mathrm{R}_{\Sigma}(b, q, \varphi)$ and $g$ be the analytic extension of $f^{-1}$ to $\mathrm{U}$ then there exists two functions $u$ and $v$, analytic in $\mathrm{U}$ with $u(0)=v(0)=0,|u(z)|<1,|v(w)|<1$ and $z, w \in U$ such that

$$
\begin{aligned}
1+\frac{1}{b}\left(\left(\tilde{D}_{q} f\right)(z)-1\right) & =\varphi(u(z)) \\
1+\frac{1}{b}\left(\left(\tilde{D}_{q} g\right)(w)-1\right) & =\varphi(v(w))
\end{aligned}
$$

where $g=f^{-1}$.

Next, define the functions $p, q \in P$ by

$$
\begin{aligned}
& p(z)=\frac{1+u(z)}{1-u(z)}=1+p_{1} z+p_{2} z^{2}+\cdots \\
& q(z)=\frac{1+v(w)}{1-v(w)}=1+q_{1} w+q_{2} w^{2}+\cdots
\end{aligned}
$$

From the above definitions, one can derive

$$
\begin{aligned}
& u(z)=\frac{p(z)-1}{p(z)+1}=\frac{1}{2} p_{1} z+\frac{1}{2}\left(p_{2}-\frac{1}{2} p_{1}^{2} z^{2}\right)+\cdots \\
& v(w)=\frac{q(w)-1}{q(w)+1}=\frac{1}{2} q_{1} w+\frac{1}{2}\left(q_{2}-\frac{1}{2} q_{1}^{2} w^{2}\right)+\cdots
\end{aligned}
$$

Combining (6), (28), (29), (32) and (33)

$$
\begin{aligned}
& 1+\frac{1}{b}\left(\left(\tilde{D}_{q} f\right)(z)-1\right)=1+\frac{1}{2} B_{1} p_{1} z+\left(\frac{1}{4} B_{2} p_{1}^{2}+\frac{1}{2} B_{1}\left(p_{2}-\frac{1}{2} p_{1}^{2}\right)\right) z^{2}+\cdots \\
& 1+\frac{1}{b}\left(\left(\tilde{D}_{q} g\right)(w)-1\right)=1+\frac{1}{2} B_{1} q_{1} w+\left(\frac{1}{4} B_{2} q_{1}^{2}+\frac{1}{2} B_{1}\left(q_{2}-\frac{1}{2} q_{1}^{2}\right)\right) w^{2}+\cdots
\end{aligned}
$$

From (34) and (35), we deduce

$$
\begin{gathered}
\frac{[2]_{q} a_{2}}{b}=\frac{1}{2} B_{1} p_{1}(36) \\
\frac{[3]_{q} a_{3}}{b}=\frac{1}{4} B_{2} p_{1}^{2}+\frac{1}{2} B_{1}\left(p_{2}-\frac{1}{2} p_{1}^{2}\right)
\end{gathered}
$$

and

$$
-\frac{[2]_{q} a_{2}}{b}=\frac{1}{2} B_{1} q_{1}
$$




$$
\frac{[3]_{q}\left(2 a_{2}^{2}-a_{3}\right)}{b}=\frac{1}{4} B_{2} q_{1}^{2}+\frac{1}{2} B_{1}\left(q_{2}-\frac{1}{2} q_{1}^{2}\right)
$$

From (36) and (38), we get

Subtracting (37) from (39) and applying (40)

$$
p_{1}=-q_{1}(40)
$$

$$
a_{3}=a_{2}^{2}+\frac{b B_{1}}{4[3]_{q}}\left(p_{2}-q_{2}\right)
$$

By adding (37) to (39), we get

Using (36) and (38)

$$
\frac{2[3]_{q}}{b} a_{2}^{2}=\frac{1}{2} B_{1}\left(p_{2}+q_{2}\right)+\frac{1}{2} p_{1}^{2}\left(B_{2}-B_{1}\right)
$$

$$
a_{2}^{2}=\frac{b B_{1}^{3}\left(p_{2}+q_{2}\right)}{4\left[b[3]_{q} B_{1}^{2}+[2]_{q}^{2}\left(B_{1}-B_{2}\right)\right]}
$$

From (41) and (42), we get

$$
a_{3}-\zeta a_{2}^{2}=b B_{1}\left[\left(h(\zeta)+\frac{1}{4[3]_{q}}\right) p_{2}+\left(h(\zeta)-\frac{1}{4[3]_{q}}\right) q_{2}\right]
$$

Where

$$
h(\zeta)=\frac{B_{1}^{2}(1-\zeta)}{4\left[b[3]_{q} B_{1}^{2}+[2]_{q}^{2}\left(B_{1}-B_{2}\right)\right]}
$$

Then, by Lemma 1 and (6)

$$
a_{3}-\zeta a_{2}^{2} \mid \leq \begin{cases}\frac{B_{1} b}{4[3]_{q}} & \text { for } 0 \$ h(\zeta) \leq \frac{1}{4[3]_{q}} \\ 4 B_{1} h(\zeta) & \text { for } h(\zeta) \geq \frac{1}{4[3]_{q}} .\end{cases}
$$

Corollary 1 If $f \in \mathrm{R}_{\Sigma}(b, q, \varphi)$ then taking $\zeta=1$, we get

$$
\left|a_{3}-a_{2}^{2}\right| \leq \frac{q^{2}|b| B_{1}}{4\left[q^{4}+q^{2}+1\right]}
$$

\section{Corollary 2 Let}

$$
\varphi(z)=\left(\frac{1+z}{1-z}\right)^{\beta}=1+2 \beta z+2 \beta^{2} z^{2}+\cdots,(0<\beta \leq 1) .
$$

then from (43), we have

\section{Corollary 3 Let}

$$
\left|a_{3}-a_{2}^{2}\right| \leq \frac{q^{2}|b| \beta}{2\left[q^{4}+q^{2}+1\right]}
$$

$$
\varphi(z)=\frac{1+(1-2 \beta) z}{1-z}=1+2(1-\beta) z+2(1-\beta) z^{2}+\cdots,(0 \leq \beta<1) .
$$

then the inequality (43) reduces to 


$$
\left|a_{3}-a_{2}^{2}\right| \leq \frac{q^{2}|b|(1-\beta)}{2\left[q^{4}+q^{2}+1\right]}
$$

\section{Conclusion}

We have estimated the bounds for the coefficients and also the linear functional which is popularly known as Fekete- Szeg $\ddot{o}$ problem, for functions belonging to the class defined in this article. We also have seen our results reducing to the results discussed in various other articles.

\section{References}

[1] H. Airault, "Symmetric sums associated to the factorization of Grunsky coefficients," In Conference, Groups and symmetries, Montreal, Canada, pp. 27-29, 2007.

[2] H. Airault, "Remarks on Faber polynomials", Int. Math. Forum, vol. 3, pp. 9-12, 2008.

[3] H. Airault and A. Bouali, "Differential Calculus on the Faber polynomials," Bull. Sci. Math., vol. 130(3), pp. 179-222, 2006.

[4] H. Airault and J. Ren, "An algebra of differential operators and generalized functions on the set of univalent function,” Bull. Sci. Math., vol. 126(5), pp. 343-367, 2002.

[5] H. Aldweby and M. A. Darus, "Subclass of harmonic univalent functions associated with q-analogue of Dziok- Srivastava operator," ISRN Math. Anal., 2013(2013), Article ID 382312, 6 pages.

[6] Altinkaya, and S. Yalçn, "The Fekete- Szegö problem for a general class of bi-univalent functions satisfying subordinate conditions," Sahand Communications in Mathematical Analysis(SCMA), vol. 5(1), pp. $1-7,2017$.

[7] Altinkaya, and S. Yalçn, "Faber polynomial coefficient estimates for a class of bi- univalent functions based on the symmetric q-derivative operator," Journal of Fractional Calculus and Applications, vol. 8(2), pp. 79-87, 2017.

[8] M. Aydoğan , Y. Kahramaner and Y. Polatoğlu, "Close-to-Convex Functions Defined by Fractional Operator," Appl. Math. Sci., vol. 7, pp. 2769-2775, 2013.

[9] K. L. Brahim and Y. Sidomou, “On some symmetric q-special functions," Le Mat., vol. 68, pp. 107-122, 2013.

[10] D. A. Brannan and J. G. Clunie, "Aspects of Contemporary Complex Analysis," Academic Press, London and New York, 1980.

[11] R. M. El-Ashwah, "Subclasses of bi-univalent functions defined by convolution," Journal of the Egyptian Mathematical Society, vol. 22(3), pp. 348-351, 2014.

[12] G. Faber, “Uber polynomische Entwickelungen,” Math. Ann. vol 57(3), pp. 389-408, 1903.

[13] B. A. Frasin, and M. K. Aouf, "New subclasses of bi-univalent functions," Applied Mathematics Letters, vol. 24, pp. 1569-1573, 2011.

[14] G. Gasper, and M. Rahman, "Basic Hypergeometric Series," Cambridge Univ. Press, Cambridge, MA, 1990. 
[15] S. G. Hamidi, and M. Jahangiri, "Faber polynomial coefficient estimates for bi-univalent functions defined by subordinations," Bull. Iranian Math. Soc., vol. 41(5), pp. 1103-1119, 2015.

[16] T. Hayami and S. Owa,"Coefficient bounds for bi-univalent functions," Panamerican Mathematical Journal, vol. 22(4), pp. 15-26, 2012.

[17] F. H. Jackson, "On q-functions and a certain difference operator," Trans. Royal Soc. Edinburgh, vol. 46, pp. 253-281, 1908.

[18] M. Lewin, "On a Coefficient problem for bi-univalent functions," Proc. Amer. Math.Soc., vol. 18, pp. 6368, 1967.

[19] A. Mohammed, and M. A. Darus, "generalized operator involving the q-hypergeometric function" Mat. Vesnik., vol. 65, pp. 454-465, 2013.

[20] H. E. Ozkan Ucar, "Cofficient inequalties for q-starlike functions," Appl. Math. Comp., vol. 276, pp. 122$126,2016$.

[21] Y. Polatoglu, "Growth and distortion theorems for generalized q-starlike functions," Adv. Math., Sci. J., vol. 5, pp. 7-12, 2016.

[22] C. Pommerenke, “Univalent Functions,”Vandenhoeck \& Ruprecht, Gottingen, 1975.

[23] S. D. Purohit and R. K. Raina, "Fractional q -calculus and certain subclass of univalent analytic functions," Mathematica, vol. 55, pp. 62-74, 2013.

[24] G. Saravanan and K. Muthunagai, "Co-efficient estimates for the class of Bi-Quasi-Convex functions using Faber polynomials,” Far East J.Math. Sci. (FJMS), vol. 102(10), pp. 2267-2276. 2017.

[25] R. Vijaya, T. V. Sudharsan and S. Sivasubramanian, "Coefficient Estimates for Certain Subclasses of Biunivalent Functions Defined by Convolution," International Journal of Analysis, Article ID 6958098,(2016),5 pages.

[26] Q. H. Xu, Y. C. Gui, and H. M. Srivastava, "Coefficient estimates for a certain subclass of analytic and bi-univalent functions,” Applied Mathematics Letters, vol. 25(6), pp. 990-994, 2012. 